\title{
Erratum: Gain-assisted superluminal propagation and rotary drag of photon and surface plasmon polaritons [Phys. Rev. A 96, 013848 (2017)]
}

\author{
Naveed Khan, Bakht Amin Bacha, Azmat Iqbal, Amin Ur Rahman, and A. Afaq \\ (Received 24 September 2017; published 13 October 2017)
}

DOI: 10.1103/PhysRevA.96.049906

A few errors were found in Eqs. (4) and (5) of our article, and those errors affected the numerical results, especially the susceptibility [Fig. 2(a)]. The values of some spectral parameters not mentioned in the paper are also provided in this Erratum.

The corrected Eq. (4), after incorporating the missing plus sign in the denominator of the first term, is as follows:

$$
\begin{aligned}
\rho_{a c}^{(2)}= & -i \Omega_{p}\left[\frac{4 \Omega_{1}^{2}\left[4\left(\gamma_{a b}-i \Delta_{p}\right)\left(i \gamma_{b d}+\Delta_{p}-\Delta_{1}\right)-i \Omega_{c}^{2}\right]}{\left(\Delta_{1}-i \gamma_{a d}\right)\left[4\left(i \gamma_{a b}+\Delta_{p}\right)\left(i \gamma_{a c}+\Delta_{p}\right)-\Omega_{c}^{2}\right] Q}\right. \\
& \left.+\frac{8 \gamma_{a d}\left(i \gamma_{a b}+\Delta_{p}\right) \Omega_{1}^{2}}{\left(\gamma_{a c}+\gamma_{a d}\right)\left(\gamma_{a d}^{2}+\Delta_{1}^{2}\right)\left(4 P+i \Omega_{c}^{2}\right)}\right]
\end{aligned}
$$

where $P=\left(\gamma_{a c}-i \Delta_{p}\right)\left(i \gamma_{a b}+\Delta_{p}\right)$ and $Q=4\left(i \gamma_{b d}+\Delta_{p}-\right.$ $\left.\Delta_{1}\right)\left(-i \gamma_{d c}-\Delta_{p}+\Delta_{1}\right)+\Omega_{c}^{2}$.

The unit of gamma $(\gamma)$ is $1 \mathrm{MHz}$ in terms of which other parameters are defined; see, for example, Ref. [1]. We have selected a moderate value of the probe field's frequency $v_{p}=$ $1000 \gamma$ as in [2]. The central or resonance frequency $\omega_{0}$ of the probe field is $970 \gamma$. In Sec. II of our paper, $v_{p}$ and $v_{1}$ are the frequencies of the probe and pump fields, respectively, instead of the phase velocities (as was written mistakenly).
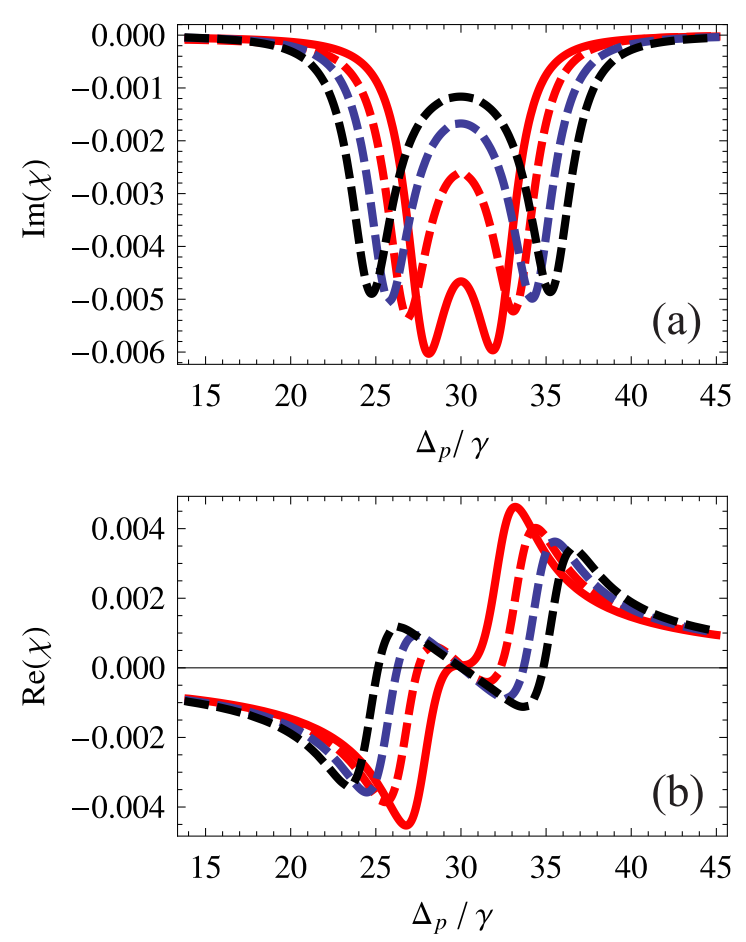

FIG. 2. Variation of (a) imaginary and (b) real part of $\chi$ with probe detuning.
The corrected Eq. (5), after fixing the typing errors, is

$$
\chi^{(2)}=\frac{3 N \lambda^{3}}{32 \pi^{3} \Omega_{p}} \rho_{a c}^{(2)},
$$

where the atomic number density is $N=5 \times 10^{12} \mathrm{~cm}^{-3}, \lambda=$ $2 \pi c / \omega$ is the wavelength of the transition between the states $a$ and $c$, while $\omega$ is the corresponding angular frequency. As usual, the susceptibility and group index plotted versus probe detuning have units of $2 N\left|\varrho_{c a}\right|^{2} / \epsilon_{0} \hbar$.

The corrected Eq. (11), after fixing the typing error, is

$$
n_{g}^{\mathrm{sp}}=\operatorname{Re}\left(n_{r}^{\mathrm{sp}}+\omega \frac{\partial n_{r}^{\mathrm{sp}}}{\partial \Delta_{p}}\right) .
$$

The concepts and conclusions of our original paper regarding the variation in susceptibility, the group index, and the rotary photon drag represented by the drag of light polarization state in the subluminal and superluminal regions remain intact.

The changes in the numerical values of the respective quantities, due to the few corrections and modified parameters, have been reflected in the revised figures in this Erratum. After incorporating the aforementioned changes, the updated plots for the susceptibility, group index, and rotary photon
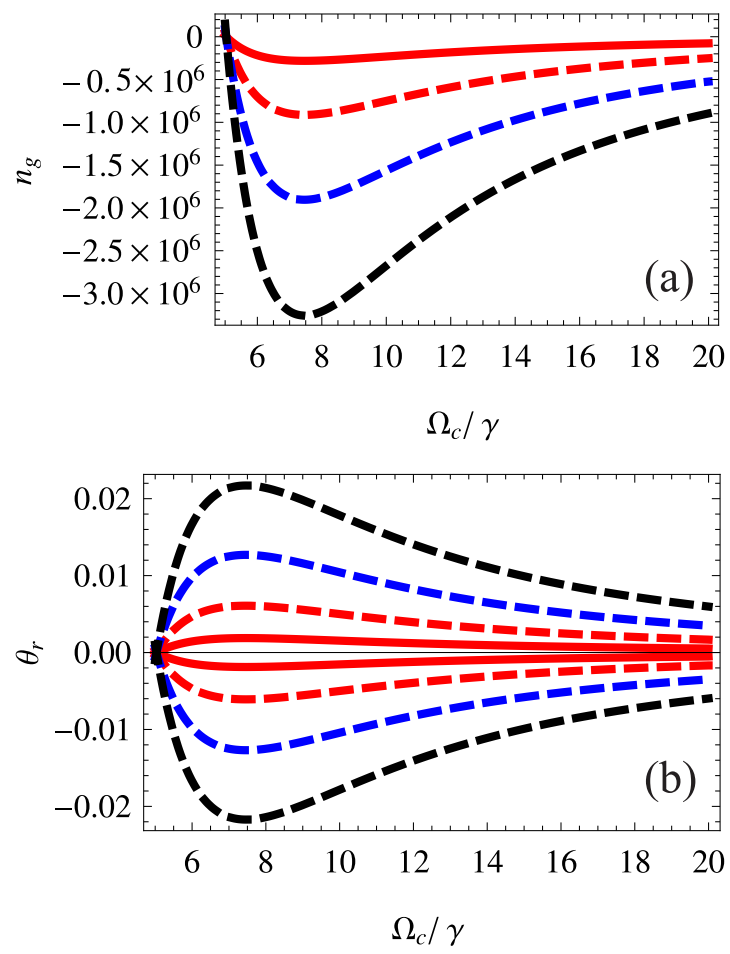

FIG. 3. Variation in (a) group index and (b) rotary photon drag with the control field's Rabi frequency $\Omega_{c}$. 


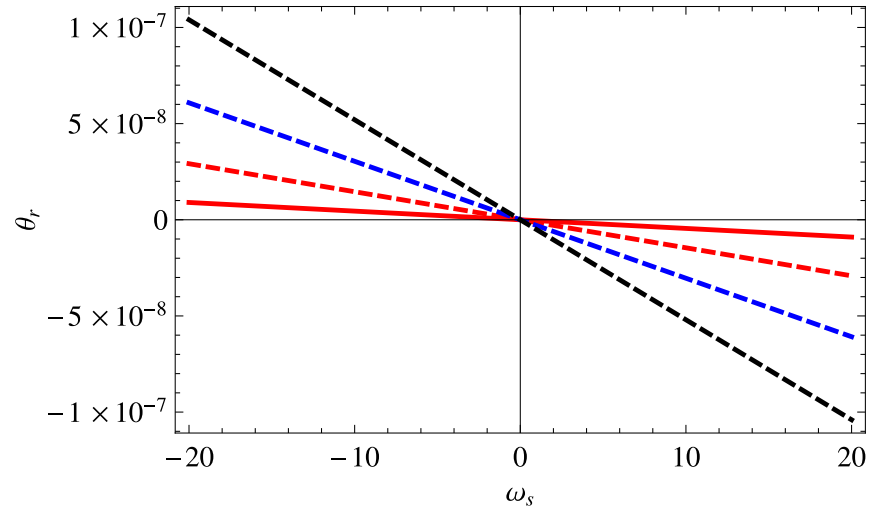

FIG. 4. Dependence of photon drag on the rotation speed of the gain-assisted atomic medium.

drag versus $\Delta_{p} / \gamma$ at the same spectral parameters of the original article, except for $\omega_{0}=970 \gamma$, are shown in the updated Figs. 2-4, respectively. The maximum (negative) value of $n_{g}$ equals $\sim 3.3 \times 10^{6}$ [Fig. 3(a)], which manifests superluminal light propagation in the gain-doublet region with a negative group velocity of magnitude $100 \mathrm{~m} / \mathrm{s}$. The corresponding photon drag is $0.022 \mathrm{rad}$. Figure 4 elucidates the variation in photon drag with respect to medium rotation velocity. The maximum value of photon drag is $\sim \mp 10^{-7} \mathrm{rad}$ at $\omega_{s}= \pm 20$ rps.
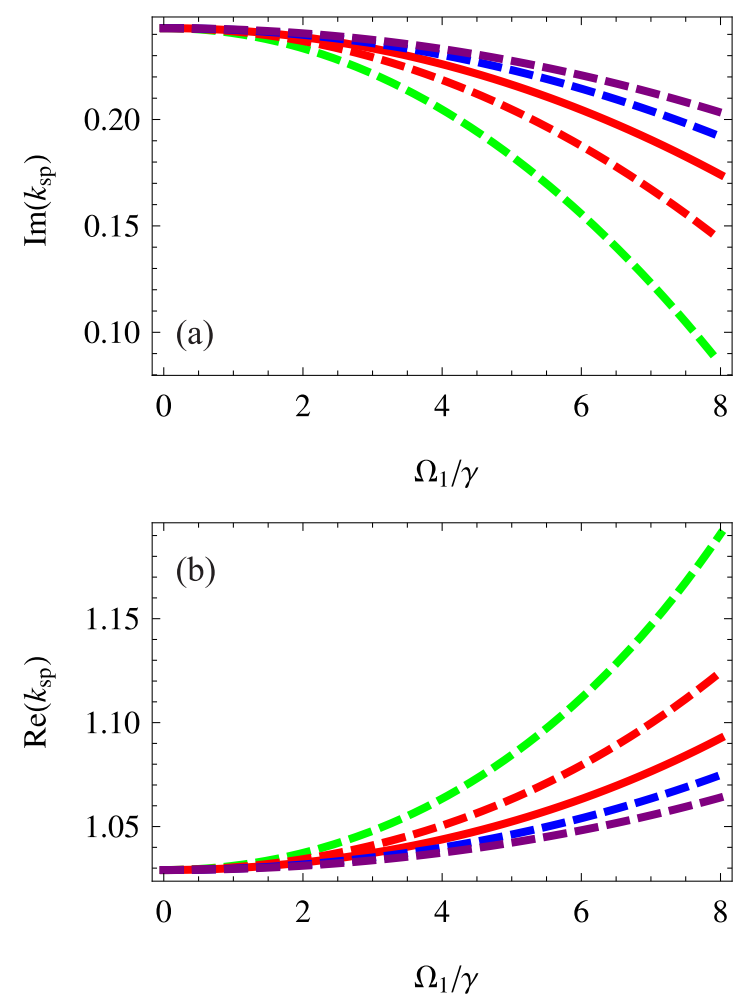

FIG. 5. Variation of (a) the imaginary part and (b) the real part of wave vector $k_{\mathrm{sp}}$ for SPPs with $\Omega_{1} / \gamma$ at fixed parameters: $\gamma_{a c, a d, b d}=$ $2.5 \gamma, \gamma_{a b, c d}=0.5 \gamma, \mu_{0}\left(\mu_{m}\right)=1,\left(\epsilon_{0}\right) \epsilon_{m}=1, \Delta_{p}=30 \gamma, \Omega_{c}=7 \gamma$ (dashed green line), $\Omega_{c}=9 \gamma$ (dashed red line), $\Omega_{c}=11 \gamma$ (solid red line), $\Omega_{c}=13 \gamma$ (dashed blue line), $\Omega_{c}=15 \gamma$ (dashed purple line), $\varphi_{1,2,3}=\pi / 2$, and $|\sigma|=2 \mathrm{~S} / \mathrm{m}$.
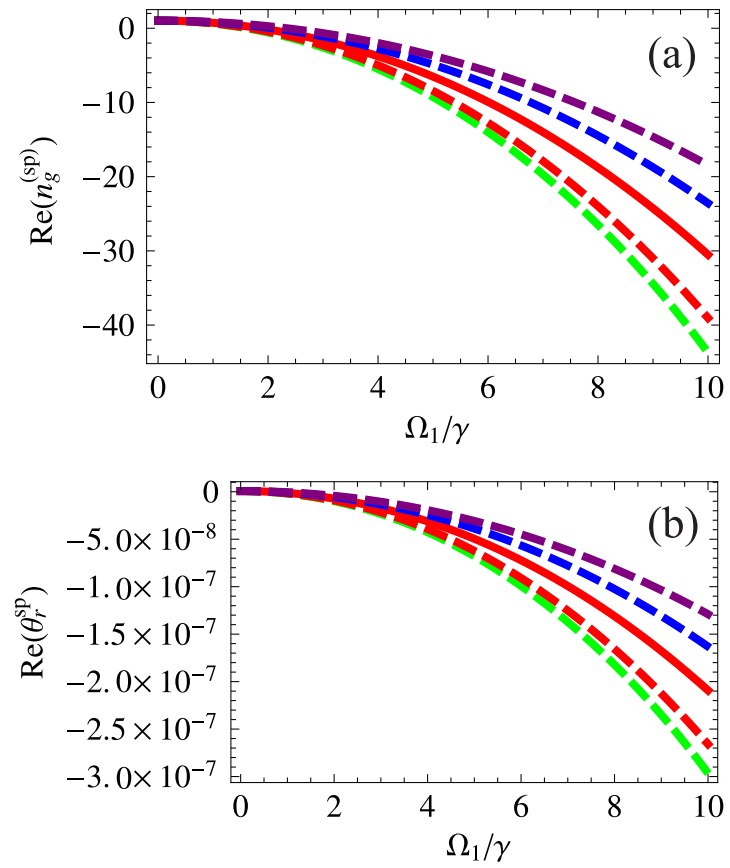

FIG. 6. Impact of $\Omega_{1} / \gamma$ on group index and the rotary SPP drag with parameters $\gamma_{a c, a d, b d}=2.5 \gamma, \gamma_{a b, c d}=0.5 \gamma, \mu_{0}\left(\mu_{m}\right)=1$, $\left(\epsilon_{0}\right) \epsilon_{m}=1, \omega_{s}=20 \mathrm{rps}, \Delta_{1, p}=30 \gamma, \varphi_{1,2,3}=\pi / 2,|\sigma|=2 \mathrm{~S} / \mathrm{m}$, $\omega_{0}=970 \gamma, \Omega_{c}=7 \gamma$ (dashed green line), $\Omega_{c}=9 \gamma$ (dashed red line), $\Omega_{c}=11 \gamma$ (solid red line), $\Omega_{c}=13 \gamma$ (dashed blue line), and $\Omega_{c}=15 \gamma$ (dashed purple line).

The modified convenient spectral parameters are stated in the captions of the corrected Figs. 5-8. It is important to note that we have plotted the physical variables of interest with respect to the pump field's Rabi frequency $\Omega_{c} / \gamma$ (varying along the $x$ axis) instead of the control field's Rabi frequency $\Omega_{1} / \gamma$. As such, the imaginary part of the wave vector $k_{\mathrm{sp}}$ of surface plasmon polaritons (SPPs) [Fig. 5(a)], which represents absorption, decreases by increasing $\Omega_{1}$ (from $0 \gamma$ to $8 \gamma$ ). However, the real part of $k_{\mathrm{sp}}$, which corresponds to SPP dispersion, increases by increasing $\Omega_{1}$. Furthermore, the increase in Rabi frequency of the control field $\Omega_{c}$ (from $7 \gamma$ to $15 \gamma$ ) corresponds to a considerable increase and decrease in the magnitude of absorption and dispersion, respectively.

Figures 6(a), 6(b), 7(a), and 7(b) demonstrate the effect of $\Omega_{1} / \gamma$ on the SPP group index $n_{g}^{\text {sp }}$ [Eq. (11)] and rotary SPP $\operatorname{drag} \theta_{r}^{\mathrm{sp}}$ [Eq. (12)] for various values of the control field's Rabi frequency $\Omega_{c}$ at fixed spectral parameters: $\Delta_{p}, \Delta_{1}, \omega_{s}, \gamma_{i j}, \sigma$, and $\omega_{0}$. Figures 6(a) and 7(a) clearly explicate a noticeable increase in $n_{g}^{\text {sp }}$ by increasing $\Omega_{1}$ for any fixed value of $\Omega_{c}$. However, an increase of $\Omega_{c}$ causes a noticeable decrease in $n_{g}^{\mathrm{sp}}$. The maximum negative value of $n_{g}^{\mathrm{sp}}$ found at $\Omega_{c}=7 \gamma$ is $\sim 43$, as shown by a dashed green curve [Figs. 6(a) and 7(a)]. This corresponds to a negative group velocity of $\sim 6.98 \times 10^{6} \mathrm{~m} / \mathrm{s}$ with $\theta_{r}^{\text {sp }} \sim \mp 3 \times 10^{-7}$ rad [Figs. 6(b) and 7(b)], respectively. The maximum drag coefficient of SPPs predicted by the model is $\sim \mp 3 \times 10^{-7}$ rad [Figs. $6(b)$ and $7\left(\right.$ b) ] at $\Omega_{1} / \gamma=10$. Moreover, the rotary SPP drag increases with an increase in $\Omega_{c}$ at a particular value of $\Omega_{1}$. The negative value of SPP drag implies that analogous to photon drag, SPP polarization state rotation also takes place opposite to the rotation of 

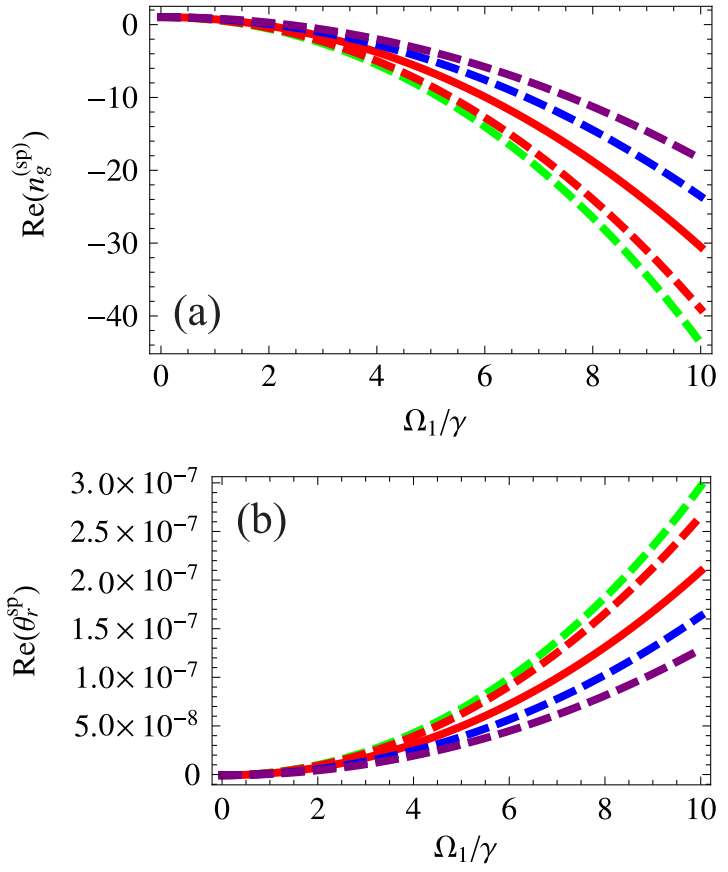

FIG. 7. Variation in the group index and the rotary SPP drag with $\Omega_{1} / \gamma$ with the parameters $\gamma_{a c, a d, b d}=2.5 \gamma, \gamma_{a b, c d}=0.5 \gamma$, $\mu_{0}\left(\mu_{m}\right)=1,\left(\epsilon_{0}\right) \epsilon_{m}=1, \omega_{s}=-20 \mathrm{rps}, \Delta_{1, p}=30 \gamma, \varphi_{1,2,3}=\pi / 2$, $|\sigma|=2 \mathrm{~S} / \mathrm{m}, \omega_{0}=970 \gamma, \Omega_{c}=7 \gamma$ (dashed green line), $\Omega_{c}=9 \gamma$ (dashed red line), $\Omega_{c}=11 \gamma$ (solid red line), $\Omega_{c}=13 \gamma$ (dashed blue line), and $\Omega_{c}=15 \gamma$ (dashed purple line).

the atomic-metal interface, which implies superluminal SPP propagation.

Figure 8 shows the dependence of SPP drag on the spin motion of the host medium. For fixed spectral parameters, the

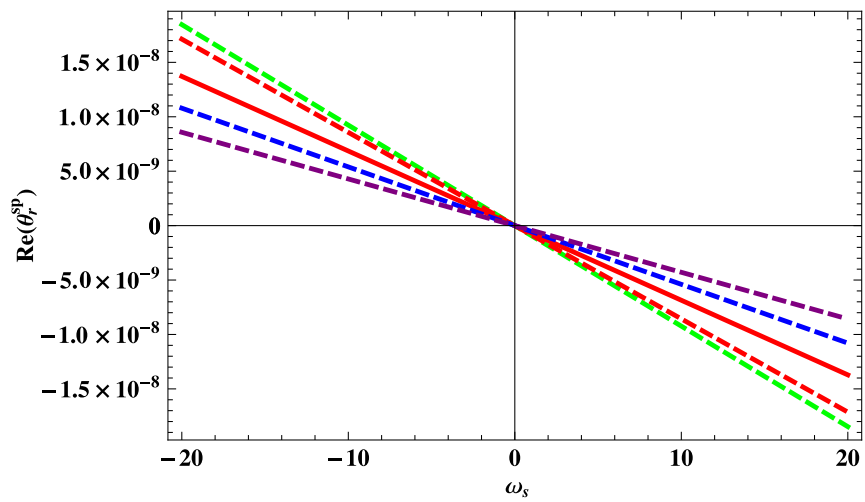

FIG. 8. Dependence of SPP drag on the rotation velocity of the atomic-metal interface and the control field's Rabi frequency such that $\gamma_{a c, a d, b d}=2.5 \gamma, \gamma_{a b, c d}=0.5 \gamma, \mu_{0}\left(\mu_{m}\right)=1,\left(\epsilon_{0}\right) \epsilon_{m}=1, \Delta_{1}=30 \gamma$, $\varphi_{1,2,3}=\pi / 2, \omega_{0}=970 \gamma, \Omega_{1}=2.5 \gamma, \Delta_{p}=30 \gamma,|\sigma|=20 \mathrm{~S} / \mathrm{m}$, $\Omega_{c}=7 \gamma$ (dashed green line), $\Omega_{c}=9 \gamma$ (dashed red line), $\Omega_{c}=11 \gamma$ (solid red line), $\Omega_{c}=13 \gamma$ (dashed blue line), and $\Omega_{c}=15 \gamma$ (dashed purple line).

SSP drag coefficient $\theta_{r}^{\mathrm{sp}}$ increases with $\omega_{s}$. In addition, an increase in the control field's Rabi frequency from $\Omega_{c}=7 \gamma$ (dashed green line) to $\Omega_{c}=15 \gamma$ (dashed purple line) results in a decrease of $\theta_{r}^{\mathrm{sp}}$. The drag coefficient has a maximum magnitude of $\sim \mp 1.8 \times 10^{-8} \mathrm{rad}$ for $\omega_{s}= \pm 20 \mathrm{rps}$ at the lowest $\Omega_{c}=7 \gamma$, and a minimum magnitude of $\sim \mp 8.6 \times 10^{-9} \mathrm{rad}$ for $\omega_{s}= \pm 20 \mathrm{rps}$ at the highest $\Omega_{c}=15 \gamma$ while keeping the other parameters constant.

The authors would like to thank Bruno Macke from the University of Lille 1 France for bringing these issues to our attention.

[1] F. Ghafoor, B. A. Bacha, and S. Khan, Phys. Rev. A 91, 053807 (2015).

[2] M. Sahrai, H. Tajalli, K. T. Kapale, and M. Suhail Zubairy, Phys. Rev. A 70, 023813 (2004). 